\title{
$3 D$ reconstruction for preoperative planning of partial anomalous pulmonary venous return
}

\author{
Alvise Guariento ${ }^{*}$, Claudia Cattapan ${ }^{1 *}$, Elisa Chemello², Francesco Bertelli', Massimo Padalino ${ }^{1}$, Elena Reffo ${ }^{3}$, \\ Giovanni Di Salvo³, Raffaella Motta², Vladimiro L Vida'
}

1Pediatric and Congenital Cardiac Surgery Unit, Department of Cardiac, Thoracic, Vascular Sciences and Public Health, University of Padua, Padua, Italy

${ }^{2}$ Institute of Radiology, Department of Medicine, University of Padua, Padua, Italy

${ }^{3}$ Pediatric Cardiology Unit, Department of Children and Woman's Health, University of Padua, Padua, Italy

*Both authors equally contributed to the study

\author{
Correspondence to: \\ Vladimiro L Vida, MD, PhD, \\ Pediatric and Congenital Cardiac \\ Surgery Unit, \\ Department of Cardiac, Thoracic, \\ Vascular Sciences and Public \\ Health, \\ University of Padua, \\ Via Giustiniani, 2-35128, Padua, \\ Italy, \\ phone: +390498212410, \\ e-mail: vladimiro.vida@unipd.it \\ Copyright by the Author(s), 2021 \\ Kardiol Pol. 2021; \\ 79 (11): 1271-1273. \\ DOI: 10.33963/KP.a2021.0135 \\ Received: \\ August 12, 2021 \\ Revision accepted: \\ October 12, 2021 \\ Published online: \\ October 13, 2021
}

\begin{abstract}
INTRODUCTION
Surgical planning in congenital heart disease (CHD) is often challenging due to the broad spectrum of conditions treated and the high variability among different individuals with the same conditions. Partial anomalous pulmonary venous return (PAPVR) represents a congenital malformation characterized by a failure of one or more pulmonary veins (PVs) to connect with the left atrium during fetal development. This event causes an anomalous communication of the right PVs, mostly the veins from the superior lobes of the right lung, with the right atrium or the superior vena cava (SVC). PAPVR is frequently associated with sinus venosus defects [1]. The anatomy of patients with PAPVR can be extremely variable and can affect the type of management [2-4].

Current standard preoperative visualization of PAPVR is generally based on cross-sectional 2D imaging [5-7], which often provides the surgeon with a suboptimal assessment due to the lack of a volumetric representation. This adversely affects the full understanding of intracardiac and vascular anatomy. More recently, the introduction of $3 \mathrm{D}$ reconstructions and modeling improved the representation of the patient's unique anatomy, which allows a more comprehensive preoperative evaluation and surgical planning, potentially reducing surgical risks [8-12].
\end{abstract}

\section{METHODS}

A retrospective analysis of patients undergoing surgical correction for PAPVR between January 2011 and December 2019 at our In- stitution, University of Padua, was performed. The study aimed to validate the utility of 3D reconstruction in planning an appropriate surgical technique in patients with PAPVR by comparing this methodology to standard 2D imaging. Patients were not identified thus consent to the processing of personal data was not obtained. The ethics committee of our institution approved data submission for publication (protocol number 4482/AO/18).

All patients with supra-diaphragmatic drainage anomalies of the right $P V s$ with suitable cardiac magnetic resonance images (MRI) were included. Exclusion criteria were the absence of preoperative imaging examines with inadequate technical characteristics for $3 \mathrm{D}$ reconstruction and patients without surgical reports. Standard hospital protocols for cardiac MRI consist of steady-state isotropic free precession sequences with electrocardiogram-gated breath-holding. The acquisition field of view ranges from the epiaortic vessels to the cranial part of the abdominal aorta.

The 3D reconstruction process was performed with Mimics InPrint 3.0 (Materialise NV, Leuven, Belgium) from bidimensional cross-sectional images. This software allows the creation of a virtual 3D model, both solid and hollow. The latter is crucial for the visualization of intracardiac and vascular anatomy. The radiologist can then perform accurate measurements directly on these virtual models (Figure 1).

A multidisciplinary team of radiologists (RM and EC) and pediatric cardiac surgeons with more than 20 years of experience in CHD 


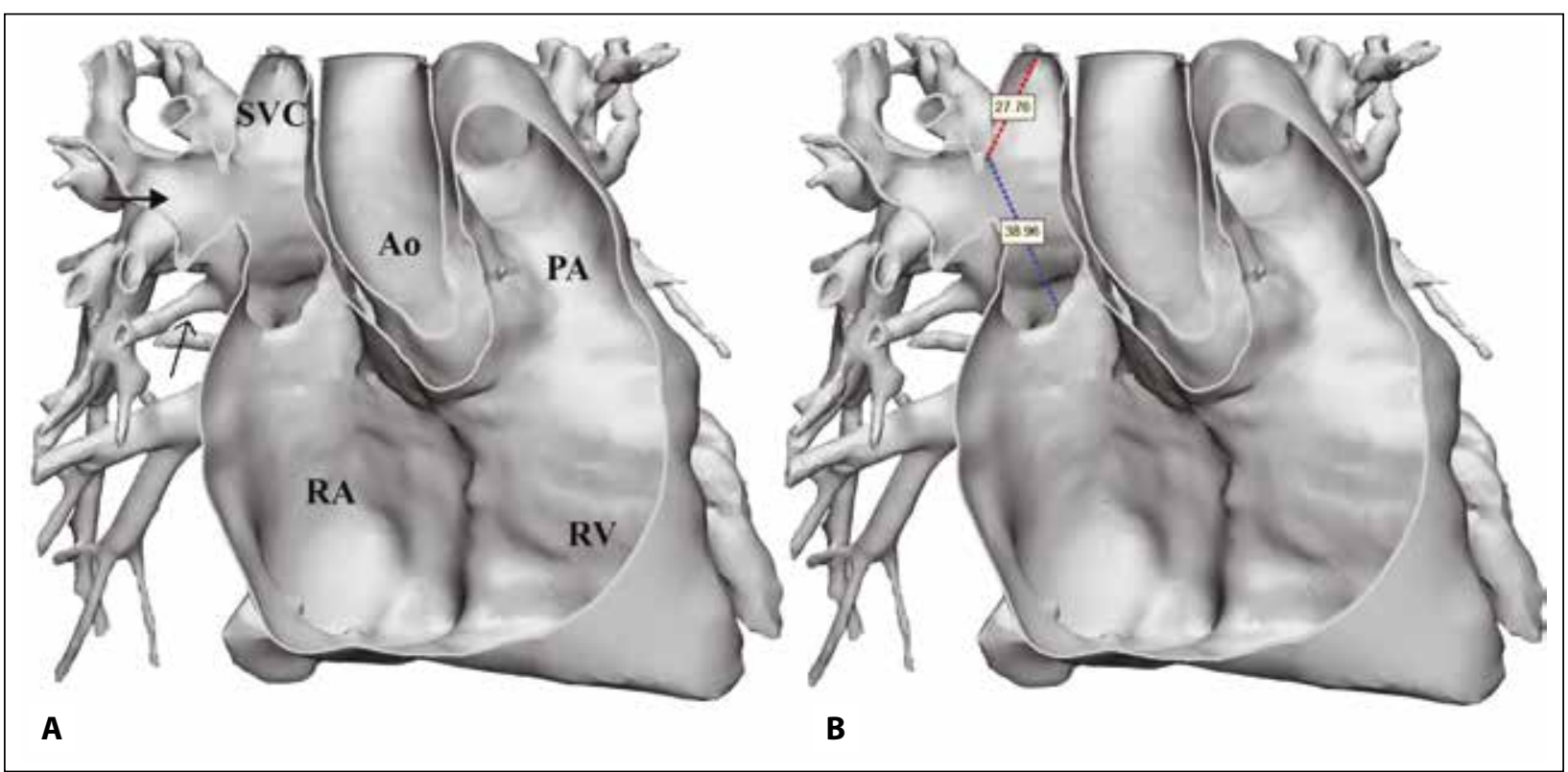

Figure 1. A stereolithographic model of the reconstructed heart. A. Arrows indicate the right middle (thin arrow) and the right upper (thick arrow) pulmonary veins. B. Measurements of the distance between the upper border of the right upper pulmonary vein and the azygos vein (red dotted line) and the inferior edge of the atrial septal communication (blue dotted line)

Abbreviations: Ao, aorta; PA, pulmonary artery; RA, right atrium; RV, right ventricle; SVC, superior vena cava

management (VV) created a list of ten parameters considered essential for adequate surgical planning in patients with PAPVR. These measures were then assessed in both standard 2D imaging and 3D reconstructions by RM and EC. Afterward, VV performed a blinded evaluation on all the measurements in order to (1) compare them with his previous operative records considered as the gold standard (performed intraoperatively directly with a silk ligature); and (2) assess the reliability of these data in relation to the actual anatomy of the patient in the operating room.

\section{Statistical analysis}

A four-point Likert scale was used to quantify the reliability and accuracy of the two methods with respect to surgical findings, using the following classes of correspondence: 4 = excellent, $3=$ good, $2=$ poor, $1=$ none. All data were analyzed using SAS (SAS Institute Inc., Cary, NC, USA). Due to the non-normal distribution, numerical variables were presented as median with interquartile range and compared using a Wilcoxon signed-rank test. The $P$-value for statistical significance was set at 0.05 .

\section{RESULTS AND DISCUSSION}

A total of 70 patients underwent surgical correction of a right PAPVR during the study period. A total of 14 patients with a median age of 8.8 (interquartile range [IQR], 4.3-12.5) years met the inclusion/exclusion criteria. A $3 \mathrm{D}$ reconstruction was performed on all 14 preoperative cardiac MRIs, and all ten measurements were obtained in each patient. The scores assigned to each parameter were analyzed and compared between 2D and 3D techniques.
The analysis showed a significant statistical improvement in six out of ten measures with the employment of $3 \mathrm{D}$ reconstruction (Supplementary material, Table S1). This demonstrated that 3D cardiac reconstruction allows for a better evaluation of PAPVR. In fact, this technology offers direct visualization of the internal anatomy of the SVC and the right atrium, thus enabling the identification of the type and dimensions of sinus venosus defects associated with PAPVR. A preoperative evaluation with 2D imaging can be more laborious for the surgeon due to the need for different sequences and scans in order to obtain a full mental picture of the patient's anatomy. Compared to $2 \mathrm{D}$ imaging, 3D reconstruction also provides better identification of the number of anomalous PVs and their spatial relationship with adjacent structures. In particular, they allow a more accurate measurement of the distance between the ostia of the anomalous PVs and the sinus venosus defect, the atrio-caval junction, and the azygos vein. Indeed, the accuracy of $2 \mathrm{D}$ imaging is limited in locating the precise position of the outlet of the PVs due to the possible overlap in the axial plane. Notably, the measurement of the distance between the anomalous PVs draining in the SVC and the sinus venosus defect was found to be the most statistically significant parameter due to the impossibility of performing this evaluation with $2 \mathrm{D}$ imaging. The localization of the two structures, in fact, lies on different and variable orthogonal planes.

This study shows how 3D reconstruction can provide an improvement in the radiological evaluation of PAPVR when comparing preoperative information with subsequent surgical findings $[11,12]$. This technology can, therefore, 
be used for personalized preoperative planning, giving cardiac surgeons an accurate spatial representation of the anatomy, as well as the ability to simulate the operation on a 3D printed model if needed [13,14].

The main limitation of this study is its retrospective design, which was necessary due to the low incidence of this disease. This undoubtedly reduced the number of 2D images available due to the lack of standardized preoperative protocols. In addition to this, a potential limitation is the use of a quality score derived from the subjective opinion of a pediatric cardiac surgeon. For this reason, the results were expressed according to a Likert scale, which is a globally recognized method for improving data standardization.

Our results confirm the important value of 3D reconstruction in the preoperative radiological assessment of $\mathrm{CHD}$ by providing additional information compared to $2 \mathrm{D}$ images. This may serve as a starting point for improving clinical and surgical management with potential personalized approaches. Further studies are needed to evaluate the impact of 3D imaging on different surgical techniques.

\section{Supplementary material}

Supplementary material is available at https://journals. viamedica.pl/kardiologia_polska.

\section{Article information}

Conflict of interests: None declared.

Open access: This article is available in open access under Creative Common Attribution-Non-Commercial-No Derivatives 4.0 International (CC BY-NC-ND 4.0) license, allowing to download articles and share them with others as long as they credit the authors and the publisher, but without permission to change them in any way or use them commercially. For commercial use, please contact the journal office at kardiologiapolska@ptkardio.pl.

How to cite: Guariento A, Cattapan C, Chemello E, et al. 3D reconstruction for preoperative planning of partial anomalous pulmonary venous return. Kardiol Pol. 2021; 79(11): 1271-1273, doi: 10.33963/KP.a2021.0135.

\section{REFERENCES}

1. Gustafson RA, Warden HE, Murray GF, et al. Partial anomalous pulmonary venous connection to the right side of the heart. JThorac Cardiovasc Surg. 1989; 98(5 Pt 2): 861-868, indexed in Pubmed: 2682021.
2. Fragata J, Magalhães $M$, Baquero $L$, et al. Partial anomalous pulmonary venous connections: surgical management. World J Pediatr Congenit Heart Surg. 2013; 4(1): 44-49, doi: 10.1177/2150135112460250, indexed in Pubmed: 23799753.

3. Kuriata J, Kwiatek P, Kołsut $P$, et al. Supracardiac partial anomalous pulmonary venous connection recognized by computed tomography in an adult patient. Kardiol Pol. 2020; 78(12): 1299-1300, doi: 10.33963/KP.15703, indexed in Pubmed: 33293499.

4. Gać P, Macek P, Dziadkowiec B, et al. Multiple atrial septal defects with concomitant partial anomalous pulmonary venous return on cardiac computed tomography. Kardiol Pol. 2020; 78(4): 348-349, doi: 10.33963/KP.15174, indexed in Pubmed: 32024810.

5. Eom HJ, Yang DH, Kang JW, et al. Preoperative cardiac computed tomography for demonstration of congenital cardiac septal defect in adults. Eur Radiol.2015;25(6): 1614-1622, doi: 10.1007/s00330-014-3547-5, indexed in Pubmed: 25519977.

6. Kilner PJ, Geva T, Kaemmerer H, et al. Recommendations for cardiovascular magnetic resonance in adults with congenital heart disease from the respective working groups of the European Society of Cardiology. Eur Heart J. 2010; 31(7): 794-805, doi: 10.1093/eurheartj/ehp586, indexed in Pubmed: 20067914.

7. Uçar T, FitozS, Tutar E, et al. Diagnostic tools in the preoperative evaluation of children with anomalous pulmonary venous connections. Int J Cardiovasc Imaging. 2008; 24(2): 229-235, doi: 10.1007/s10554-007-9246-4, indexed in Pubmed: 17597422.

8. Greil GF, Wolf I, Kuettner A, et al. Stereolithographic reproduction of complex cardiac morphology based on high spatial resolution imaging. Clin Res Cardiol. 2007; 96(3): 176-185, doi: 10.1007/s00392-007-0482-3, indexed in Pubmed: 17225916.

9. Biglino G, Capelli C, Wray Jo, et al. 3D-manufactured patient-specific models of congenital heart defects for communication in clinical practice: feasibility and acceptability. BMJ Open. 2015; 5(4): e007165, doi: 10.1136/bmjopen-2014-007165, indexed in Pubmed: 25933810.

10. Mitsouras D, Liacouras $P$, Imanzadeh A, et al. Medical 3D printing for the radiologist. Radiographics. 2015; 35(7): 1965-1988, doi: 10.1148/rg.2015140320, indexed in Pubmed: 26562233.

11. Cattapan C, Bertelli F, Guariento A, et al. 3D ultrasound-based fetal heart reconstruction: a pilot protocol in prenatal counselling. Rev Esp Cardiol (Engl Ed). 2021; 74(6): 549-551, doi: 10.1016/j.rec.2020.11.002, indexed in Pubmed: 33281102.

12. Raimondi F, Vida V, Godard C, et al. Fast-track virtual reality for cardiac imaging in congenital heart disease. J Card Surg. 2021;36(7): 2598-2602, doi: 10.1111/jocs.15508, indexed in Pubmed: 33760302.

13. Sodian R, Weber S, Markert M, et al. Stereolithographic models for surgical planning in congenital heart surgery. Ann Thorac Surg. 2007; 83(5): 1854-1857, doi: 10.1016/j.athoracsur.2006.12.004, indexed in Pubmed: 17462413.

14. Yoo SJ, Spray T, Austin EH, et al. Hands-on surgical training of congenital heart surgery using 3-dimensional print models. J Thorac Cardiovasc Surg. 2017; 153(6): 1530-1540, doi: 10.1016/j.jtcvs.2016.12.054, indexed in Pubmed: 28268011. 